УДК 532.516

\title{
ЧИСЛЕННОЕ МОДЕЛИРОВАНИЕ ТЕПЛООБМЕНА И ГИДРОДИНАМИКИ В КРУГЛОЙ ТРУБЕ С НАКЛОННО-ТАНГЕНЦИАЛЬНОЙ ЗАКРУТКОЙ ПОТОКА НА ВХОДЕ
}

\author{
Халатов А.А., ${ }^{1,3}$, академик НАН Украины, Кобзарь С.Г., канд. тех. наук, Дашевский Ю.Я. \\ ${ }^{1}$ Институт технической теплофизики НАН Украины, ул. Желябова, 2а, Киев, 03680, Украина \\ ${ }^{2}$ ГП НПКГ «Зоря»-«Машпроект», пр. Октябрьский, 42a, Николаев, 54018, Украина \\ ${ }^{3}$ Национальный технический университет Украины «КПИ», Проспект Победь 37, Киев, 03056, \\ Украина
}

Використовуючи пакет прикладних програм ANSYS CFX виконано комп'ютерне моделювання гідродинаміки та теплообміну в круглій трубі 3 нахильно-тангенціальним завихренням потоку на вході та $90^{\circ}$ поворотом на виході. Виконано верифікацію двох груп моделей турбулентності, визначені основні параметри розрахункової сітки, які дозволяють отримати прийнятну точність розрахунків. Співставлення результатів розрахунків 3 експериментальними даними показало, що використання $k-\omega$ моделі турбулентності дає кращі результати при розрахунку поверхневого кута завихрення потоку; всі моделі турбулентності добре розраховують коефіцієнт гідравлічного опору, а $L R R$ модель напружень Рейнольдса найбільш точно описує теплообмін.
С использованием пакета прикладных программ ANSYS CFX выполнено компьютерное моделирование гидродинамики и теплообмена в круглой трубе с наклоннотангенциальной закруткой потока на входе и $90^{\circ}$ поворотом на выходе. Выполнена верификация двух групп моделей турбулентности, определены основные параметры расчетной сетки, позволяющие получить приемлемую точность расчета. Сопоставление результатов расчетов с экспериментальными данными показало, что использование $k$ - $\omega$ модели турбулентности дает наилучшие результаты при расчете поверхностного угла закрутки потока; все модели турбулентности хорошо описывают коэффициент гидравлического сопротивления, а $L R R$ модель напряжений Рейнольдса наиболее точно описывает теплообмен.
Based on the commercial software ANSYS CFX the computer simulation of heat transfer and hydrodynamics was performed in the round tube with incline-tangential flow swirl at the inlet and flow $90^{\circ}$ turn at the exit. Two groups of turbulence models and basic parameters of the computer grid were verified allowing obtain the accepted accuracy. Comparison of predicted and experimental results showed that $k-\omega$ turbulence model provides good agreement for the surface swirl angle, all tested models describes well the pressure drop coefficient, while LRR model of Reynolds stresses predicts precisely heat transfer data.

Библ. 10, табл. 4, рис. 6.

Ключевые слова: гидродинамика, закрученный поток, теплоотдача, наклонно-тангенциальная закрутка; модели турбулентности.

$d$ - диаметр цилиндрического канала, м;

$f$ - коэффициент гидравлического сопротивления;

$k-$ кинетическая энергия турбулентности;

$P$ - давление, Па;

$y^{+}-$безразмерное расстояние от стенки до первой

ячейки расчетной сетки;

$T$ - температура, $\mathrm{K},{ }^{\circ} \mathrm{C}$;

$u, v, w$ - компоненты скорости потока по осям $x$, $y, z, \mathrm{M} / \mathrm{c}$;

\section{Введение}

Закрученные течения широко применяют в технических устройствах для интенсификации процессов теплообмена и совершенствования $x, y, z$ - оси декартовой системы координат;

$\alpha$ - коэффициент теплоотдачи, Вт/ $\mathrm{M}^{2} \mathrm{~K}$;

$\phi-$ угол закрутки потока, градус;

$v$ - кинематический коэффициент вязкости, $\mathrm{M}^{2} / \mathrm{c}$;

$\rho-$ плотность, кг/ $\mathrm{M}^{3}$;

$\varepsilon-$ турбулентная диссипация;

$\mathrm{Nu}$ - число Нуссельта;

$\mathrm{Re}$ - число Рейнольдса.

многих теплофизических процессов. Подавляющее большинство опубликованных исследований выполнены для условий классической тангенциальной закрутки потока, когда поток на 
входе в трубу подается под углом $90^{\circ}$ к ее продольной оси. В последние годы в связи с разработкой и исследованием внутреннего циклонного охлаждения лопаток газовых турбин самостоятельный интерес получила наклоннотангенциальная закрутка, когда поток подается тангенциально и под углом к оси канала менее $90^{\circ}$ в направлении движения потока. Большой цикл экспериментальных исследований теплообмена и гидродинамики в этом направлении представлен в работе [1]. Полученные результаты кроме самостоятельного значения могут служить основой для разработки более точных методов компьютерного моделирования закрученного потока с использованием современных коммерческих пакетов.

Цель и задачи исследования. С использованием коммерческого программного комплекса Ansys CFX выполнить компьютерное моделирование гидродинамики и теплообмена в круглой трубе с наклонно-тангенциальной закруткой потока и $90^{\circ}$ поворотом на выходе. На основе сравнения с результатами экспериментального исследования $[1,9,10]$ определить адекватные модели турбулентности и получить новые данные в области, прилегающей к наклонно-тангенциальному завихрителю.

\section{Объект исследования и компьютерная модель}

В качестве объекта исследований использован круглый канал с внутренним диаметром 20 мм (рис. 1), исследованный экспериментально в работе [1]. Исследуемая геометрия была построена в декартовой системе координат. При компьютерном моделировании использовалась неструктурированная комбинированная расчетная сетка, построенная при помощи сеточного генератора ANSYS CFX Mesh., которая представляет собой комбинацию тетраэдральных элементов в области основного потока с призматическими элементами в области сгущения расчётной сетки около твёрдой поверхности канала. Согласно рекомендациям разработчика программы ANSYS CFX, количество слоев призматических элементов около стенки должно быть не меньше 10. На предварительной стадии было выполнено исследование влияния способа задания сгущения сетки около твердой стенки канала на ее качество. Перебор методов создания сгу-
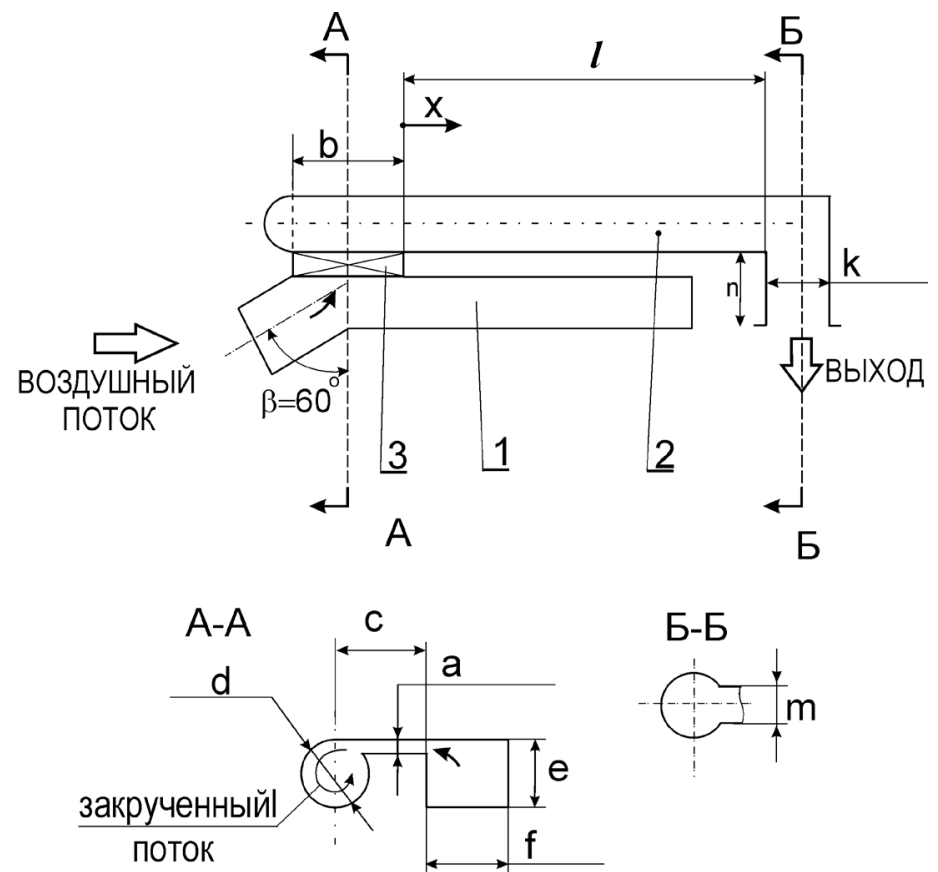

Б-Б

Рис. 1. Геометрическая модель объекта исследования [1]: 1 - распределительный канал; 2 - тангенциальный завихритель;

3 -основной цилиндрический канал.

Основные размеры: $a=5 \mathrm{mм} ; b=59 \mathrm{mм}$; $c=30 \mathrm{Mm} ; d=20 \mathrm{~mm} ; e=20 \mathrm{~mm} ; f=25 \mathrm{~mm}$; $k=40 \mathrm{Mm} ; l=231 \mathrm{~mm} ; m=15 \mathrm{mM} ; n=40 \mathrm{MM}$.

щения сетки около стенки канала показал, что наиболее оптимальным способом задания сгущения является метод Максимальная толщина слоя, причем значение максимальной толщины должно быть равным 0,0005 м $(0,025$ диаметра канала). Исследуемая геометрия построена в среде ANSYS Design Manager, общий вид 3D компьютерной модели показан на рис. 2.

Анализ опубликованных данных показал [27], что для расчета закрученного течения в круглом канале хорошие результаты демонстрируют две группы моделей турбулентности. Первая из них включает модели $k-\varepsilon, R N G k$ - $\varepsilon$ и $L R R$ модель напряжений Рейнольдса, использующие масштабируемые пристеночные функции. При использовании этой группы в расчетах применялась сетка с параметрами, которые приведены в таблице 1 , причем значение $y^{+}$не превышало 40. Вторая группа моделей турбулентности состоит из моделей $k-\omega$ и $S S T$, которые используют автоматические пристеночные функции. 
Табл. 1. Параметры расчетной сетки

\begin{tabular}{|c|c|c|c|}
\hline \multirow{2}{*}{\multicolumn{2}{|c|}{ Параметры сетки }} & \multicolumn{2}{|c|}{ Класс моделей турбулентности } \\
\hline & & $\begin{array}{c}\text { Масштабируемые } \\
\text { пристеночные } \\
\text { функции } \\
\end{array}$ & $\begin{array}{c}\text { Автоматические } \\
\text { пристеночные } \\
\text { функции } \\
\end{array}$ \\
\hline \multirow{3}{*}{$\begin{array}{l}\text { Сгущение } \\
\text { около стенки }\end{array}$} & Максимальная толщина слоя, м & 5,0 e-4 & 5,0 e-4 \\
\hline & Количество слоев, ед. & 15 & 17 \\
\hline & Фактор роста & 1,2 & 1,3 \\
\hline \multicolumn{2}{|c|}{ Максимальный размер ячейки на поверхностях, м } & 2,0 e-3 & 2,0 e-3 \\
\hline \multicolumn{2}{|c|}{ Максимальный размер ячейки, м } & 2,0 e-3 & 2,0 e-3 \\
\hline \multicolumn{2}{|c|}{ Количество элементов } & 808325 & 874863 \\
\hline \multicolumn{2}{|c|}{ Количество узлов } & 1694651 & 1861029 \\
\hline
\end{tabular}

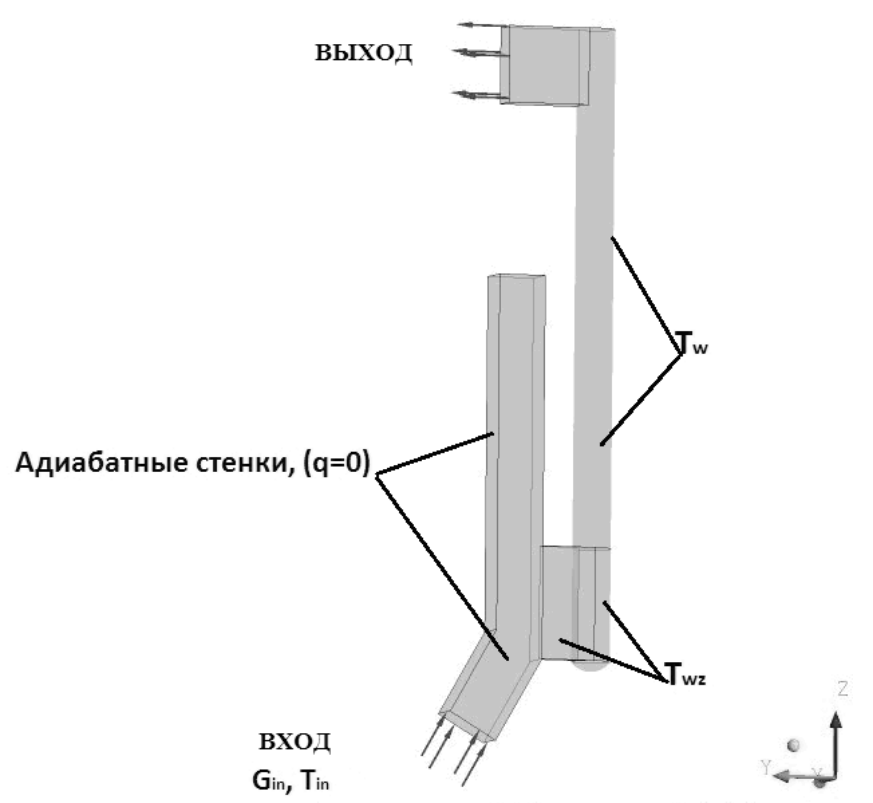

\section{Рис. 2. Компьютерная 3D-модель объекта исследования и схема постановки граничных условий.}

Для данной группы использовалась расчетная сетка с параметрами, которые приведены в таблице 1 , при этом значение $y^{+}$не превышало 1,0 .

\section{Физическая модель и граничные условия}

При анализе моделей турбулентности были использованы результаты экспериментов по гидродинамике $[1,9]$ и теплообмену $[1,10]$ закрученного потока в трубе с наклонно-тангенциальной закруткой потока и $90^{\circ}$ поворотом на выходе, которые характеризуются высокой степенью по- вторяемости. Рассмотрен случай теплообмена от нагретого воздушного закрученного потока к охлаждаемой стенке. Среднерасходное число Рейнольдса в обоих случаях составляло 100000, что практически соответствует реальным условиям внутреннего охлаждения лопаток газовых турбин.

Схема постановки граничных условий задачи представлена на рис. 2, перечень граничных условий приводится в таблице 2. На выходе из расчетной области (за выходным поворотом) задавалось атмосферное статическое давление равное 101325 Па.

Табл. 2. Граничные условия

\begin{tabular}{|l|c|}
\hline Расход воздуха $G_{i n}$, $/ \mathrm{c}$ & 32,28 \\
\hline Температура воздуха $T_{i n},{ }^{\circ} \mathrm{C}$ & 97 \\
\hline Температура стенки завихрителя $T_{w z},{ }^{\circ} \mathrm{C}$ & 30 \\
\hline $\begin{array}{l}\text { Температура стенки цилиндрического } \\
\text { канала } T_{w},{ }^{\circ} \mathrm{C}\end{array}$ & 20 \\
\hline
\end{tabular}

Воздух считался идеальным газом с теплофизическими свойствами, зависящими от температуры. В частности, зависимость теплоёмкости при постоянном давлении от температуры задавалась полиномиальной зависимостью вида [8]:

$\frac{C_{p}}{R}=a_{o}+a_{1} \cdot T+a_{2} \cdot T^{2}+a_{3} \cdot T^{3}+a_{4} \cdot T^{4}$,

где $R$ - универсальная газовая постоянная; $a_{0} \ldots$ $a_{4}-$ коэффициенты полинома [8]. 
Зависимость коэффициента динамической вязкости и теплопроводности воздуха от температуры задавались формулами Сазерленда [8]:

$\mu=\mu_{0}\left(\frac{T}{T_{0}}\right)^{n} \frac{T_{o}+C_{S}}{T+C_{S}}, \quad \lambda=\lambda_{0}\left(\frac{T}{T_{0}}\right)^{n} \frac{T_{o}+C_{S 1}}{T+C_{S 1}}$,

где $\mu_{0}, \lambda_{0}-$ базовые значения коэффициентов динамической вязкости и теплопроводности соответственно; $T_{0}=273,15 \mathrm{~K}$ базовое значение температуры воздуха.

Осреднённые по Рейнольдсу уравнения Навье-Стокса решались для вязкого теплопроводного газа в стационарной постановке, при расчете теплообмена использовано полное уравнение сохранения энергии.

\section{Результаты моделирования}

Как указывалось выше, при компьютерном моделировании были использованы две группы моделей турбулентности - модели с автоматическими пристеночными функциями (SST и $k-\omega)$ и с масштабируемыми пристеночными функциями $(k-\varepsilon, R N G k-\varepsilon, L R R$ модель напряжений Рейнольдса - LRR-RSM). Сравнение расчетных и экспериментальных результатов проводилось по углу закрутки потока вблизи стенки канала, коэффициенту гидравлического сопротивления и числу Нуссельта $\mathrm{Nu}_{d}$.

Угол закрутки потока в цилиндрическом канале определялся путем обработки рассчитанного поля скорости в CFX-post, при помощи созданной пользовательской переменной. Для декартовой системы координат тангенс угла закрутки определялся согласно формуле:

$\operatorname{tg} \varphi=\frac{\sqrt{u^{2}+v^{2}}}{\sqrt{w^{2}}}$.

Коэффициент гидравлического сопротивления определялся соотношением:

$f=\frac{\Delta P^{*}}{\Delta x} d \frac{2}{\rho w_{c p}{ }^{2}}$,

где $w_{\text {ср }}$ - среднерасходная осевая скорость потока в канале. При использовании стандартной функции CFX-post для определения коэффициента теплоотдачи, в случае использования моделей турбулентности с автоматическими пристеноч- ными функциями, результаты расчета числа Нуссельта получаются завышенными в несколько раз. В этом случае целесообразно использовать экспертный параметр $T_{b u l k}$, который используется в функции CFX-post в качестве определяющей температуры потока. Также возможен вариант расчета осредненного коэффициента теплоотдачи, используя рассчитанный компьютерной программой тепловой поток и коэффициент теплоотдачи:

$\alpha=$ [areaAve(Wall Heat Flux)@User Surface 1] / [areaAve(Temperature)@Plane 1-

- areaAve(Temperature)@User Surface 1],

здесь: Plane 1 - выбранное поперечное сечение верхность расчетной области, которая образована пересечением поперечного сечения Plane 1 со стенкой канала.

Результаты расчета изменения поверхностного угла закрутки потока, коэффициента гидравлического сопротивления и локального числа Нуссельта по длине канала представлены на рис. 3 - 6. Начало отсчета соответствует выходному «срезу» тангенциальной щели, направление оси $x$ показано на рис. 1.

На рис. 3 приведены результаты расчета и экспериментальных измерений поверхностного угла закрутки потока $[1,9]$. Как следует, аппроксимированное на начало координат значение этого угла составляет около $50^{\circ}$, тогда как его геометрическое значение равно $30^{\circ}$ (рис. 1). Это обусловлено перестройкой потока еще до входа потока в щель тангенциального завихрителя.

Сравнение показывает (рис. 3), что в области $x / d>2,0$ все модели с автоматическими и масштабируемыми пристеночными функциями достаточно хорошо описывают изменение этого параметра, но наилучшие результаты показывает $k$ - $\omega$ модель турбулентности. Некоторая неопределенность в определении угла закрутки имеет место в области $0<x / d<1,5 \ldots 2,0$, где наблюдается значительное расхождение результатов расчета. В первом приближении можно принять, что наиболее адекватные результаты демонстрирует $S S T$ - модель турбулентности, данные которой при $x=0$ соответствуют экспериментальным значениям угла закрутки, аппроксимированным 


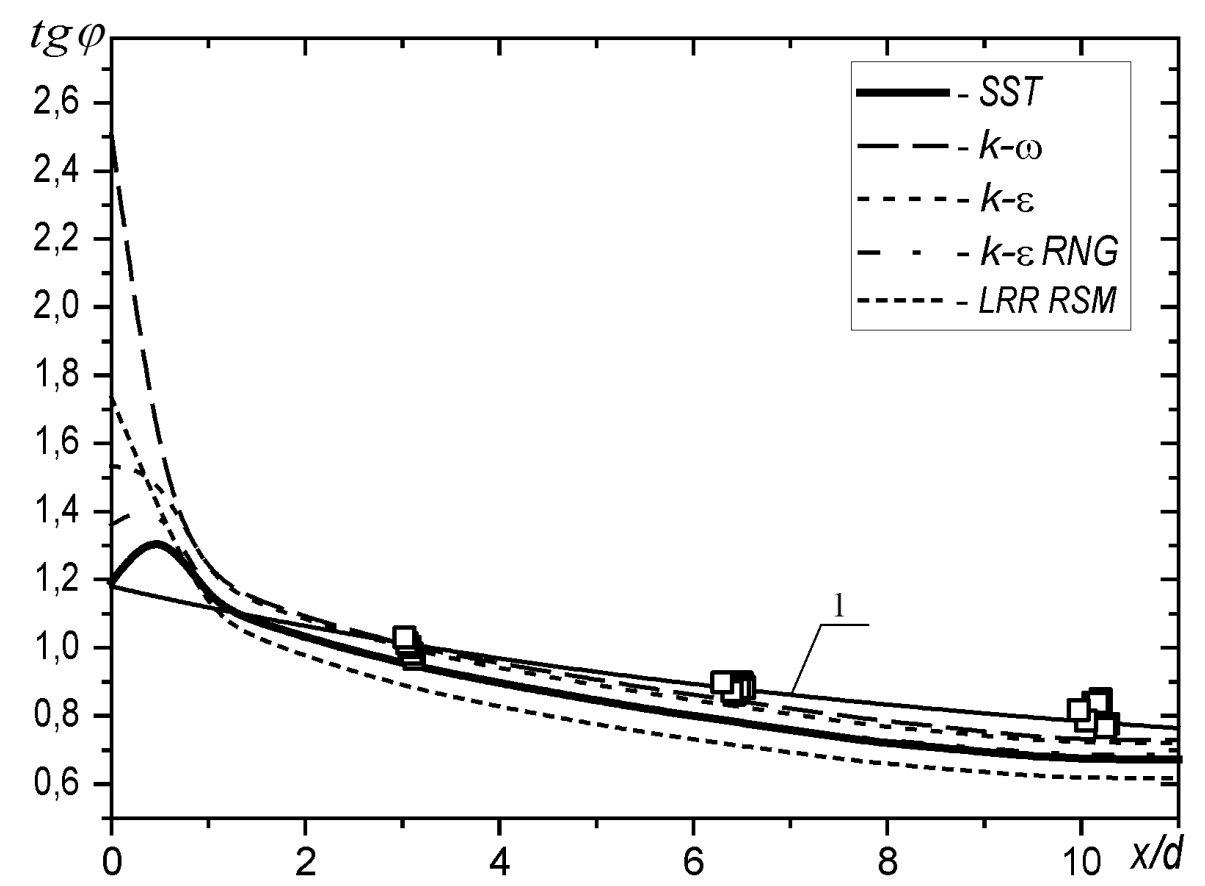

Рис. 3. Продольное изменение значения тангенса угла закрутки: 口-эксперимент [9]; 1 - кривая аппроксимации экспериментальных данных [9].

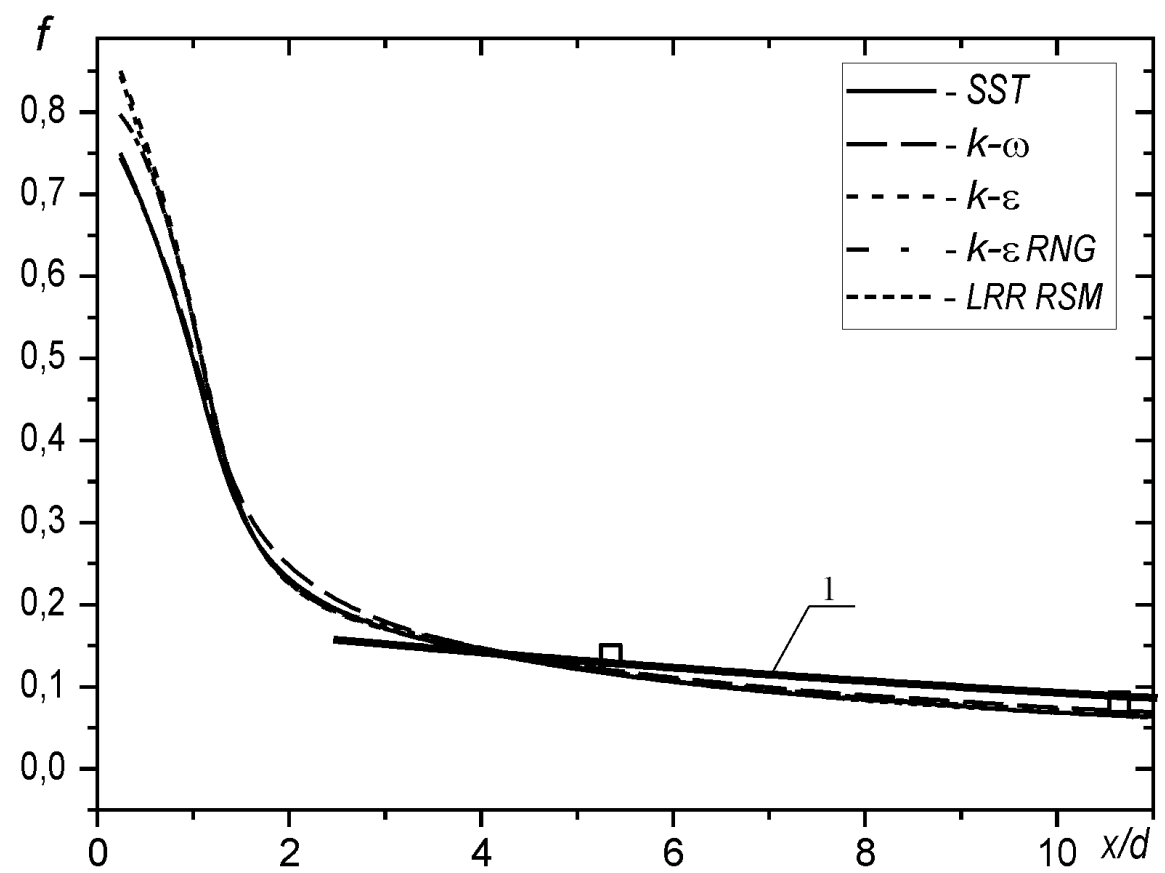

Рис. 4. Продольное распределение коэффициента гидравлического сопротивления: 口- эксперимент [9]; 1 - кривая аппроксимации экспериментальных данных [9].

на начало координат. Что касается экстремального поведения этой кривой в области $x / d<1,5$, то это может быть обусловлено перестройкой структуры потока внутри канала.

На рис. 4 представлено сравнение расчетных и экспериментальных данных по коэффициенту гидравлического сопротивления. В области $x / d>3,0$ все модели турбулентности дают примерно одинаковые результаты и хорошо согласуются с экспериментальными данными [9] и 
аппроксимирующей кривой полученной в работе [9]. В области $x / d<3,0$ наблюдаются значительный рост потерь полного давления, что объясняется перестройкой структуры потока в области сразу после завихрителя. В качестве коэффициента гидравлического сопротивления $f$ при $x=0$ можно принять среднее значение равное 0,85 .
На рис. 5, 6 представлены результаты расчета локального числа Нуссельта. Для моделей с автоматическими пристеночными функциями расчет выполнен с использованием экспертного параметра $T_{\text {bulk, }}$, который используется пакетом $C F X$ в качестве определяющей температуры потока (при расчетах этот параметр задавался рав-

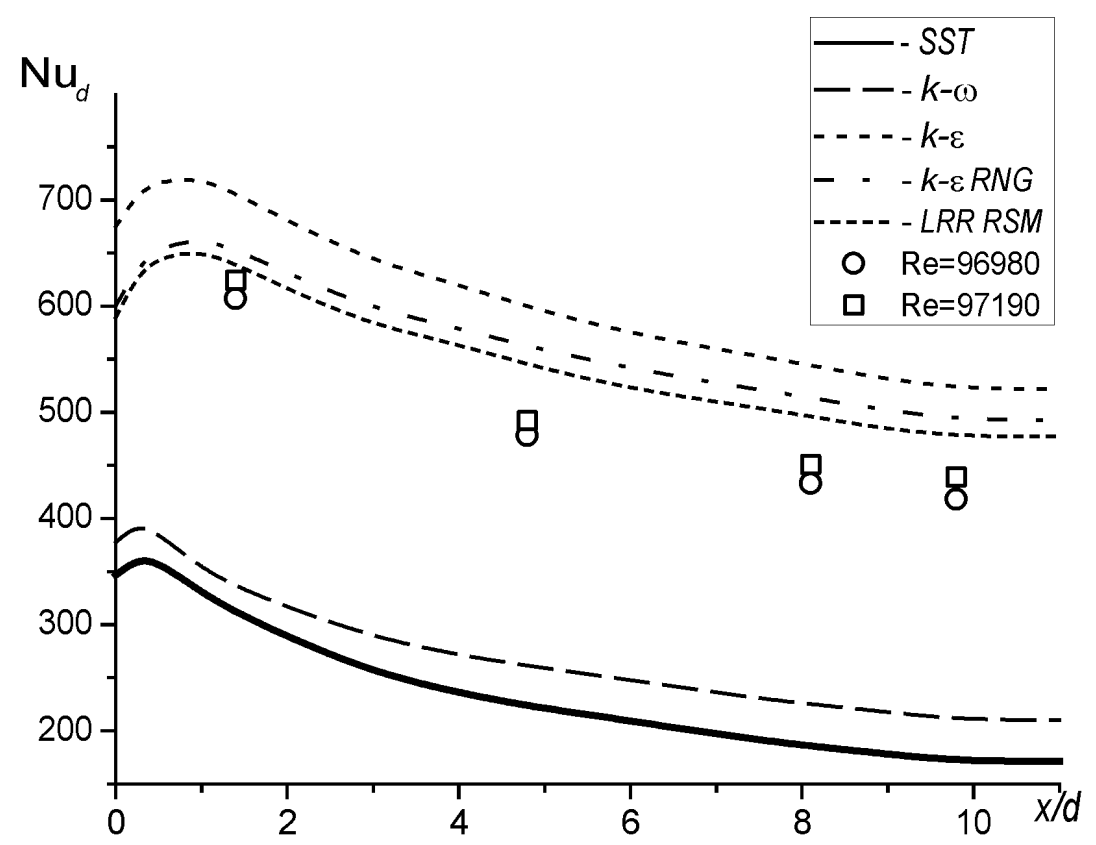

Рис. 5. Продольное распределение локального числа Нуссельта. Точки - экспериментальные данные работы [10].

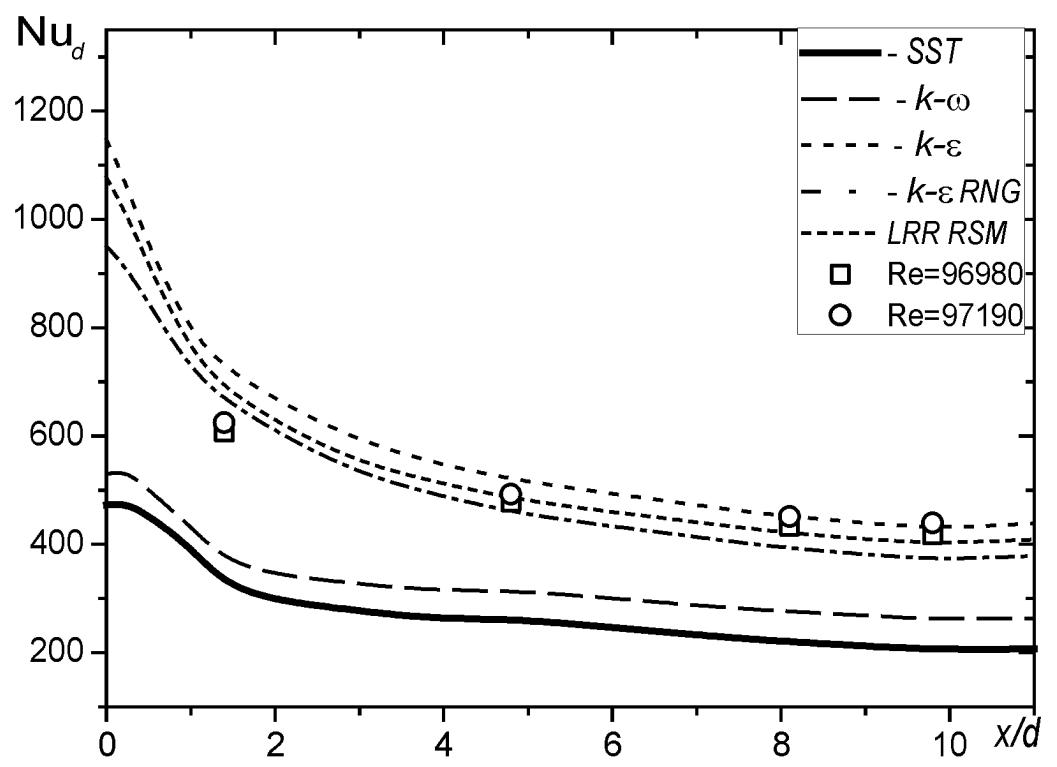

Рис. 6. Продольное распределение локального числа Нуссельта. Точки - экспериментальные данные работы [10]. 
ным средней температуре потока в трубе). Для моделей с масштабируемыми пристеночными функциями число Нуссельта рассчитывалось по коэффициенту теплоотдачи, полученному стандартными средствами $C F X$-post.

Анализ результатов приведенных на рис. 5, показывает, что для закрученного потока в трубе модели турбулентности с автоматическими пристеночными функциями дают существенно заниженное значение числа Нуссельта, в то время как модели с масштабируемыми пристеночными функциями ( $R N G k-\varepsilon ; L R R R S M)$ показывают удовлетворительную точность.

На рис. 6 приведены результаты расчета числа Нуссельта с использованием коэффициента теплоотдачи рассчитанного по выражению (3). Применение такого подхода позволило повысить точность расчета теплоотдачи с использованием моделей с автоматическими пристеночными функциями, но достигнутая точность является не удовлетворительной. В тоже время результаты расчета теплоотдачи, которые получены с использованием модели напряжений Рейнольдса $(L R R-R S M)$ дают хорошее согласование с экспериментальными данными в области $x / d>1,5$. Полученные результаты также показывают значительный рост теплообмена в области $x / d<1,5$.

\section{Потери в завихрителе и в выходном повороте}

Компьютерное моделирование позволило определить гидравлические потери в наклоннотангенциальном завихрителе, а также в выходном $90^{\circ}$ повороте. Коэффициент гидравлического сопротивления завихрителя определялся соотношением:

$$
\xi_{z}^{*}=\frac{\Delta P^{*}}{\rho_{u} \frac{\bar{w}_{u}{ }^{2}}{2},}
$$

где $\Delta P^{*}$ - разность полных давлений перед завихрителем и в сечении трубы за завихрителем на расстоянии $x / d=1,5$ от выходного «среза» тангенциальной щели (как это было принято в работе [8]); $w_{\text {ш }} \rho_{\text {ш }}-$ среднемассовая скорость и плотность воздуха в щели завихрителя.

Коэффициент гидравлического сопротивления выходного поворота определялся по соотношению:

$$
\xi_{t u m}^{*}=\frac{\Delta P^{*}{ }_{t u m}}{\rho_{c p} \frac{w_{c p}{ }^{2}}{2}},
$$

где $\Delta P_{\text {tum }}^{*}-$ разность полных давлений перед поворотом и на выходе из него; $w_{\mathrm{cp}}, \rho_{\mathrm{cp}}$ - среднемассовая скорость потока в канале и плотность в цилиндрическом канале на входе в поворот.

Результаты сравнения коэффициента гидравлического сопротивления завихрителя при использовании различных моделей турбулентности представлены в таблице 3. Эти данные показывают, что наилучшее согласование расчета с экспериментом демонстрирует $L R R$ модель напряжений Рейнольдса. Относительная погрешность расчета составляет 9,4 \%. Что касается потерь полного давления в самой щели тангенциального завихрителя, то, используя данные, представленные на рис. 4 и в таблице 2, можно получить, что коэффициент гидравлического сопротивления $\zeta_{z}^{* *}$ составляет 2,01. Здесь $\zeta_{z}^{* *}=$ $=2 \Delta P_{\text {щ }}^{*} /\left(\rho_{\text {щ }} w_{\text {щ }}^{2}\right)$.

Результаты расчета коэффициента гидравлического сопротивления выходного поворота представлены в таблице 4. Эти данные показывают, что наилучшее согласование расчетных данных с экспериментом получено при использовании $k$ - $\omega$ модели турбулентности. Относительная погрешность расчета в этом случае составляет 9,14 \%. В целом, более высокая погрешность

\begin{tabular}{|c|c|c|c|}
\hline Модель турбулентности & Расчет & Эксперимент [8] & Относительная погрешность, \% \\
\hline$k-\varepsilon$ & 2,757 & \multirow{5}{*}{3,086} & 10,6 \\
\hline$k-\varepsilon-R N G$ & 2,754 & & 10,75 \\
\hline$k-\omega$ & 2,711 & & 12,1 \\
\hline$S S T$ & 2,775 & & 10,07 \\
\hline LRR-RSM & 2,796 & & 9,4 \\
\hline
\end{tabular}

Табл. 3. Результаты расчета коэффициента сопротивления завихрителя 
Табл. 4. Результаты расчета коэффициента сопротивления выходного поворота $\zeta_{\text {turn }}^{*}$

\begin{tabular}{|c|c|c|c|}
\hline Модель турбулентности & Расчет & Эксперимент [8] & Относительная погрешность, \% \\
\hline$k-\varepsilon$ & 0,8384 & \multirow{5}{*}{0,703} & 19,2 \\
\hline$k-\varepsilon-R N G$ & 0,8655 & & 23 \\
\hline$k-\omega$ & 0,7673 & & 9,14 \\
\hline$S S T$ & 0,7925 & & 12,7 \\
\hline$L R R-R S M$ & 0,796 & & 13,2 \\
\hline
\end{tabular}

расчета коэффициента сопротивления выходного поворота, может быть обусловлена различием в задании величины статического давления на выходе из расчетной области и статического давления на выходе из участка поворота, наблюдаемого в экспериментах. Эти данные в работе [9] не приводятся.

\section{Выводы}

1. С использованием пакета прикладных программ ANSYS CFX выполнено компьютерное моделирование гидродинамики и теплообмена в трубе с наклонно-тангенциальной закруткой потока и $90^{\circ}$ поворотом на входе. В расчетах использованы две группы моделей турбулентности - с автоматическими пристеночными функциями (SST и $k$ - $\omega$ модели) и с масштабируемыми пристеночными функциями $(k-\varepsilon$, $R N G k-\varepsilon, L R R-R S M$ модели).

2. Определены параметры расчетной сетки, при которых пакет прикладных программ $A N S Y S$ $C F X$ позволяет получить приемлемую точность расчета гидродинамики и теплообмена в трубе с наклонно-тангенциальной закруткой потока.

3. Сопоставление результатов расчета с экспериментальными данными показало, что:

- наилучшие результаты при расчете поверхностного угла закрутки потока в области $x / d>2,0$ дает использование $k$-ш модели турбулентности;

- все модели турбулентности показывают примерно одинаковые и хорошо согласующиеся с экспериментом результаты расчета гидравлического сопротивления в области $x / d>3,0$;

- наилучшие результаты при расчете локального теплообмена во всей области канала получены при использовании $L R R$ модели напряжений Рейнольдса;

- при расчете коэффициента гидравличес- кого сопротивления наклонно-тангенциального завихрителя наилучшее согласование с экспериментом демонстрирует $L R R$ модель напряжений Рейнольдса, а при расчете коэффициента гидравлического сопротивления выходного поворота $k$ - $\omega$ модель турбулентности.

4. Получены новые данные, характеризующие некоторые особенности гидродинамики и теплообмена около наклонно-тангенциального завихрителя.

\section{ЛИТЕРАТУРА}

1. Теплообмен и гидродинамика при циклонном охлаждении лопаток газовых турбин/ Халатов А.А., Романов В.В., Борисов И.И., Дашевский Ю.Я., Северин С.Д. / Изд. Института технической теплофизики НАН Украины. - Киев. - 2010. - 317 с.

2. John P.C.W. Ling, Peter T. Ireland, Neil. W. Harvey. Measurement of Heat Transfer Coefficient Distributions and Flow Field in a Model of a Turbine Blade Cooling Passage with Tangential Injection // ASME Paper \# GT2006-90352.

3. Zhao LIU, Zhen Ping FENG, Liming Song. Numerical Study on Flow and Heat Transfer Characteristics of Swirl Cooling on Leading Edge Model of Gas Turbine Blade // ASME Paper \# GT201146125.

4. Hay, N., \& West, P.D., 1975, "Heat Transfer in Free Swirling Flow in a Pipe," ASME Journal of Heat Transfer, P. $411-416$.

5. Andreas Lerch, Heinz-Peter Schiffer, Daniela Klaubert. Impact on Adiabatic Film Cooling Effectiveness Using Internal Cyclone Cooling // ASME Paper GT2011-45120.

6. D.W. Stephens, K. Mohanarangam. Turbulence model analysis of flow inside a hydrocyclone // Seventh International Conference on CFD in the Minerals and Process Industries CSIRO, Melbour- 
ne, Australia. - 9-11 December, 2009.

7. Ali M. Jawarneh, Hitham Tlilan, Ahmad AlShyyab, Amer Ababneh. Strongly swirling flows in a cylindrical separator//Minerals Engineering 21 (2008)/ P. $366-372$.

8. Introduction to Thermal Science/Shmidt F., Henderson R., Wolgemuth C./ John Wiley\&Son. New York. - 1984. - 445 p.

9. Халатов А.А., Борисов И.И., Дашевский Ю.Я., Северин С.Д. Гидродинамика закручен-

\section{NUMERICAL SIMULATION OF HEAT TRANSFER AND HYDRODYNAMICS IN ROUND TUBE WITH INCLINED- TANGENTIAL FLOW SWIRL AT THE INLET}

ного потока в трубе с наклонно-тангенциальной закруткой потока и 90-поворотом на выходе // Промышленная теплотехника. - 2009. - Т. 31, №6. - C. $6-13$.

10. A. Khalatov, I. Borisov, S. Severin, V. Romanov, V. Spitsyn, Yu. Dashevskyy. Heat Transfer, Hydrodynamics and Pressure Drop in the Model of a Blade Leading Edge Cyclone Cooling // Proc. of ASME Turbo Expo 20011. - GT2011-45150. - 9 p. models describes well the pressure drop coefficient, while $L R R$ model of Reynolds stresses predicts precisely heat transfer data. References 10 , tables. 4 , figures 6 .

Key words: hydrodynamics, swirling flow, heat transfer, inclined-tangential flow swirl, turbulence models.

1. Heat transfer and hydrodynamics at cyclone cooling of gas turbine blades / Khalatov A.A., Romanov V.V., Borisov I.I., Dashevskyy Yu.Y., Severin S.D. / Institute for Engineering Thermophysics. National Academy of Sciences of Ukraine. - Kyiv. 2010. - 317 p. (Rus.)

2. John P.C.W. Ling, Peter T. Ireland, Neil. $W$. Harvey. Measurement of Heat Transfer Coefficient Distributions and Flow Field in a Model of a Turbine Blade Cooling Passage with Tangential Injection // ASME Paper \# GT2006-90352.

3. Zhao LIU, Zhenping FENG, Liming Song. Numerical Study on Flow and Heat Transfer Characteristics of Swirl Cooling on Leading Edge Model of Gas Turbine Blade // ASME Paper \# GT2011-46125.

4. Hay, N., \& West, P. D. Heat Transfer in Free Swirling Flow in a Pipe// ASME Journal of Heat 
Transfer. - 1975. - P. $411-416$.

5. Andreas Lerch, Heinz-Peter Schiffer, Daniela Klaubert. Impact on Adiabatic Film Cooling Effectiveness Using Internal Cyclone Cooling // ASME Paper GT2011-45120.

6. D.W. Stephens, K. Mohanarangam. Turbulence model analysis of flow inside a hydrocyclone // Seventh International Conference on CFD in the Minerals and Process Industries CSIRO, Melbourne, Australia. - 9-11 December 2009.

7. Ali M. Jawarneh, Hitham Tlilan, Ahmad Al-Shyyab, Amer Ababneh. Strongly swirling flows in a cylindrical separator//Minerals Engineering. 2008. - v.21. - P. $366-372$.
8. Introduction to Thermal Science/Shmidt F., Henderson R., Wolgemuth C./ John Wiley\&Son.New York. -1984 . $-445 \mathrm{p}$.

9. A. Khalatov, I. Borisov, Yu. Dashevsky, S. Severin. Hydrodynamics of the swirled $\mathrm{fl}$ ow in the tube with the obliquely-tangential fl ow swirl and 90 degrees outlet channel bend // Promyshlennaya teplotekhnika. - 2009. - vol. 31, № 6. P. 6 - 13. (Rus.)

10. A. Khalatov, I.Borisov, S. Severin, V. Romanov, V. Spitsyn, Yu. Dashevskyy. Heat Transfer, Hydrodynamics and Pressure Drop in the Model of a Blade Leading Edge Cyclone Cooling // Proc. of ASME Turbo Expo 20011. - GT2011-45150. - 9 p.

Получено 05.06.2014 Received 05.06.2014 